\title{
Construction and Expression of Mutant cDNAs Responsible for Genetic Polymorphism in Aldehyde Oxidase in Donryu Strain Rats
}

\author{
Mayuko Adachi ${ }^{1}$, Kunio Itoh ${ }^{1}$, Akiko Masubuchi ${ }^{1}$, Nobuaki Watanabe ${ }^{2}$ and Yorihisa Tanaka,* \\ ${ }^{1}$ Department of Drug Metabolism and Pharmacokinetics, Tohoku Pharmaceutical University, Sendai, Japan \\ ${ }^{2}$ Drug Metabolism and Pharmacokinetics Research Laboratories, Daiichi-Sankyo Co. Ltd., Tokyo, Japan
}

Received 18 May 2007, Accepted 22 August 2007

\begin{abstract}
We demonstrated the genetic polymorphism of aldehyde oxidase (AO) in Donryu strain rats: the ultrarapid metabolizer (UM) with nucleotide mutation of (377G, 2604C) coding for amino acid substitution of (110Gly, 852 Val), extensive metabolizer (EM) with (377G/A, 2604C/T) coding for (110Gly/Ser, 852 Val/Ala), and poor metabolizer (PM) with (377A, 2604T) coding for (110Ser, 852Ala), respectively. The results suggested that $377 \mathrm{G}>\mathrm{A}$ and/or $2604 \mathrm{C}>\mathrm{T}$ should be responsible for the genetic polymorphism. In this study, we constructed an $E$. coli expression system of four types of AO cDNA including Mut-1 with (377G, 2604T) and Mut-2 with (377A, 2604C) as well as naturally existing nucleotide sequences of UM and PM in order to clarify which one is responsible for the polymorphism. Mut-1 and Mut-2 showed almost the same high and low activity as that of the UM and PM groups, respectively. Thus, the expression study of mutant AO cDNA directly revealed that the nucleotide substitution of $377 \mathrm{G}>\mathrm{A}$, but not that of $2604 \mathrm{C}>\mathrm{T}$, will play a critical role in the genetic polymorphism of $\mathrm{AO}$ in Donryu strain rats. The reason amino acid substitution will cause genetic polymorphism in AO activity was discussed.
\end{abstract}

Keywords: Aldehyde oxidase, Donryu rat, Expression, Mutant, Single nucleotide polymorphism (SNP)

\section{Introduction}

Aldehyde oxidase (AO, EC 1.2.3.1) is a major member of the molybdenum hydroxylase family along with xanthine oxidase. Both enzymes consist of a homodimer with a subunit molecular mass of about $150 \mathrm{kDa}$. Each subunit contains a molybdopterin

*To whom correspondence should be addressed. Tel: 81-22-727-0108; Fax: 81-22-275-2013

E-mail: ytanaka@tohoku-pharm.ac.jp cofactor, a FAD, and two different $2 \mathrm{Fe}-2 \mathrm{~S}$ redox centers that are essential for catalytic activity. AO catalyzes the oxidation of a wide range of endogenous and exogenous aldehydes and $N$-heterocyclic aromatic compounds. The representative $N$ heterocyclic drugs that serve as substrates for $\mathrm{AO}$ are methotrexate, 6-mercaptopurine, cinchona alkaloids, and famciclovir (Beedham, 1985, 1987, 1997, 2002; Kitamura et al., 2006). In addition, AO can catalyze in vitro reduction of a variety of functional groups including sulfoxides, $\mathrm{N}$-oxides, azo dyes, and $N$-hydroxycarbamoyl substituents in the presence of an appropriate electron donor (Kitamura et al., 2006). In fact, the atypical antipsychotic drug, ziprasidone, is mostly metabolized to its reductive ring-cleaved $S$-methyldihydroziprasidone by AO in human (Prakash et al., 1997; Beedham et al., 2003). Marked species differences have been well documented for the AO-catalyzed metabolism of drugs including methotrexate (Kitamura et al., 1999a; Jordan et al., 1999) and famciclovir (Rashidi et al., 1997). A large variation in rat strains has also been demonstrated in the oxidation activity of benzaldehyde (Sugihara et al., 1995) and methotrexate (Kitamura et al., 1999b). In addition, an interesting individual difference has been reported in Wistar rats (GlueksonWaelsch et al., 1967) and in SD rats (Beedham, 1998).

RS-8359, $( \pm$ )-4-(4-cyanoanilino)-5,6-dihydro-7-hydroxy-7Hcyclopenta $[d]$-pyrimidine], is a reversible and selective MAOA inhibitor (Yokoyama et al., 1989; Kumagae et al., 1991; Miura et al., 1993; Iwata et al., 1996), and has been developed as an antidepressant (Puchler et al., 1997; Plenker et al., 1997). One of the major metabolic pathways of RS-8359 is 2oxidation on the pyrimidine ring to give the 2-keto metabolite. Cytosolic AO was identified as a responsible enzyme for the stereospecific formation of 2-keto metabolite from the $(S)$ enantiomer. Similar to the already reported findings regarding AO, we observed remarkable species differences, strain differences in rats, and individual differences in Donryu strain rats in the AO-catalyzed metabolism of RS-8359 (Takasaki et al., 2005; Itoh et al., 2005, 2006, 2007). Monkeys and humans have an extremely high $\mathrm{AO}$ activity that results in a substantially 
non-detectable plasma concentration of the $(S)$-enantiomer accompanied by a high rate of excretion of the 2-keto metabolite in urine. There was a 230-fold variation of $V_{\max }$ values between the highest activity in the Wistar-Imamichi strain rat and the lowest activity in the Slc-Wistar strain rat (Sasaki et al., 2006). The AO-catalyzed 2-oxidation activity of (S)-RS-8359 in Donryu strain rats was divided into three groups: ultrarapid metabolizer (UM), extensive metabolizer (EM), and poor metabolizer (PM). Nucleotide sequence analysis revealed that there were only two nucleotide mutations among them, namely UM with (377G, 2604C), EM with (377G/A, 2604C/T), and PM with (377A, 2604T) (Itoh et al., 2007).

In the current study, we constructed an E. coli expression system of two mutant AO cDNAs of Donryu strain rat, Mut-1 with $(377 \mathrm{G}, 2604 \mathrm{~T})$ and Mut-2 with $(377 \mathrm{~A}, 2604 \mathrm{C})$, and characterized their enzyme activity to directly reveal which of the mutations---377G $>$ A or $2604 \mathrm{C}>$ T---is more critical in maintaining catalytic activity.

\section{Materials and Methods}

Chemicals and reagents. The $(S)$-enantiomer of RS-8359 and its 2-keto metabolite were supplied by Ube Kosan Co. Ltd. Hydrocortisone, an internal standard of HPLC analysis, was purchased from Sigma Chemical Co. Imidazole was obtained from Wako Pure Chemical Industries, Ltd. All other reagents were of reagent grade.

\begin{abstract}
Animals. Seven-week-old Crj:Donryu strain rats were purchased from Charles River Japan. The animals were housed according to the Guidelines for Animal Experimentation (Tohoku Pharmaceutical University) in cages in rooms with a unidirectional airflow at a controlled temperature $\left(22 \pm 2^{\circ} \mathrm{C}\right)$, relative humidity $(50 \pm 10 \%)$, and 12-h light/dark cycles (07.00-19.00 h). Tap water was available ad libitum and CE2 food (Clea Japan) was available ad libitum except for overnight fasting before use.
\end{abstract}

Enzyme activity assay. (S)-RS-8359 $(100 \mu \mathrm{M})$ was incubated at $37^{\circ} \mathrm{C}$ for $60 \mathrm{~min}$ in a reaction mixture $(0.25 \mathrm{ml})$ consisting of 100 $\mathrm{mM}$ phosphate buffer ( $\mathrm{pH} 6.0), 1.0 \mathrm{mM} \mathrm{K}{ }_{3} \mathrm{Fe}(\mathrm{CN})_{6}, 0.13 \mathrm{mM}$ EDTA, and the expressed protein $(5 \sim 20 \mu \mathrm{g} / \mathrm{ml}, 0.10 \mathrm{ml})$. The reaction was stopped by the addition of acetonitrile $(0.50 \mathrm{ml})$ containing $0.2 \mathrm{mg} / \mathrm{ml}$ of hydrocortisone as an internal standard. The mixture was then centrifuged at $10,000 \mathrm{~g}$ for $5 \mathrm{~min}$. Aliquots $(25$ $\mu \mathrm{l})$ of the supernatant were analyzed for quantification of the oxidation product by reversed-phase HPLC on a YMC ODS A-312 column ( $6.0 \mathrm{~mm}$ i.d. $\times 150 \mathrm{~mm}$, YMC Co. Ltd.). The mobile phase was composed of acetonitrile $/ 0.5 \%$ ammonium acetate (25:75); the flow rate was $1.0 \mathrm{ml} / \mathrm{min}$. The HPLC instrument was a Shimadzu model 6A High Performance Liquid Chromatograph System (Shimadzu Seisakusho Co. Ltd.). The peaks were monitored for absorbance at $315 \mathrm{~nm}$; the peak area was calculated on a Chromatopac C-R4A (Shimadzu Seisakusho).

Preparation of anti-rat liver AO antisera. Rabbit anti-rat liver AO antisera used in this study were previously prepared by Sasaki et al. (2006). Briefly, the purified AO (1.0 mg protein $/ \mathrm{ml})$ from the liver cytosol of Wistar rats was emulsified with an equal volume of Freund's complete adjuvant (Sigma). Each of two rabbits was immunized with $1 \mathrm{ml}$ of immunogen by intradermal injection every 4 weeks. Four months after the first immunization, blood was taken by cardiac puncture and antisera were prepared by a conventional method. Pooled antisera were stored at $-80^{\circ} \mathrm{C}$ until used for Western blot analysis.

Direct sequence analysis of genomic DNA. Genomic DNA was isolated from liver samples with a DNeasy Blood \& Tissue Kit (QIAGEN) according to the manufacturer's instructions. Primer sets were designed in reference to the nucleotide sequences of rat (GenBank accession number NW 047816) on introns as follows: 377F:5'-gaggtcttctctccaaattcagtg-3', 377R:5'-ggcatatggtaggtgtccaatt atta-3', 2604F:5'-cttcccaatatcactaaaccagcta-3', 2604R:5'-gcagtgagcc ctttattagatgtta-3'. The PCR reaction was conducted in a $50-\mu 1$ solution containing $1 \times$ Ex-Taq buffer, $0.2 \mathrm{mM}$ dNTP mix solution, 2.5U Ex-Taq DNA polymerase (TaKaRa Bio Inc.), $2 \mathrm{mM} \mathrm{MgCl}$, $1 \mu \mathrm{M}$ each of forward and reverse primer, and $2 \mu \mathrm{l}$ of the template. PCR amplification was conducted using a PCR thermal cycler MP (TaKaRa) as follows: initial denaturation at $94^{\circ} \mathrm{C}$ for $5 \mathrm{~min}$, and 30 cycles of $94^{\circ} \mathrm{C}$ for $30 \mathrm{~s}, 60^{\circ} \mathrm{C}$ for $30 \mathrm{~s}, 72^{\circ} \mathrm{C}$ for $30 \mathrm{~s}$, and a final extension at $72^{\circ} \mathrm{C}$ for $10 \mathrm{~min}$. The PCR products obtained were purified using the Wizard SV Gel and PCR Clean-Up System (Promega Co.). The DNA sequences were determined using a CEQ 8000 Genetic Analysis System (Beckman Coulter Inc.) and a DTCS Quick Start Kit, for which the above-mentioned forward or reverse primer was used as the direct sequence primer.

Construction of expression plasmids for UM, PM, and mutant AO of Donryu strain rats The cDNAs of UM and PM of AO in Donryu strain rats have previously been subcloned in XL-TOPO vector using the TOPO TA Cloning Kit (Invitrogen) in this laboratory (Itoh et al., 2006). The preserved XL-TOPO plasmids of $\mathrm{AO}$ were now used to construct the expression system for the two types of polymorphic AO, UM and PM, as follows. The XL-TOPO plasmids $(20 \mu \mathrm{g})$ were first digested with $\mathrm{NgoMIV}$ (New England Biolabs) followed by double digestion with Acc65I and SalI (New England Biolabs). pQE-30 Xa vector (20 $\mu$ g, QIAGEN) was also double digested with the same restriction enzymes, and then treated with Bacterial Alkaline Phosphatase (TaKaRa). The AO cDNA and cleaved pQE-30 Xa vector were purified with Wizard SV Gel and PCR Purification Kit and TE buffer (Promega). The cleaved pQE30 Xa was ligated with AO cDNA of UM or PM using a TaKaRa DNA Ligation Kit Ver. 2 (TaKaRa) according to the manufacturer's protocol. The resulting plasmids were transfected into $E$. coli XL1Blue MRF' (TaKaRa), and the insertion of AO cDNA of UM or PM into the plasmids was checked by agarose gel electrophoresis after digestion with $\mathrm{SacI}$ for $2 \mathrm{~h}$ at $37^{\circ} \mathrm{C}$.

The construction strategies for the mutant plasmids of $\mathrm{AO}$ in Donryu strain rats are shown in Fig. 1. Both plasmids containing AO cDNA of UM and PM were treated separately with HpaI and then with Acc65I for $18 \mathrm{~h}$ at $37^{\circ} \mathrm{C}$. Small cDNA fragments containing nucleotide mutation at 377 and a large one containing that at 2604 were purified by agarose gel electrophoresis. Ligation was conducted in the same manner as described above in such a way that mutant plasmid Mut-1 had the nucleotide sequences of 


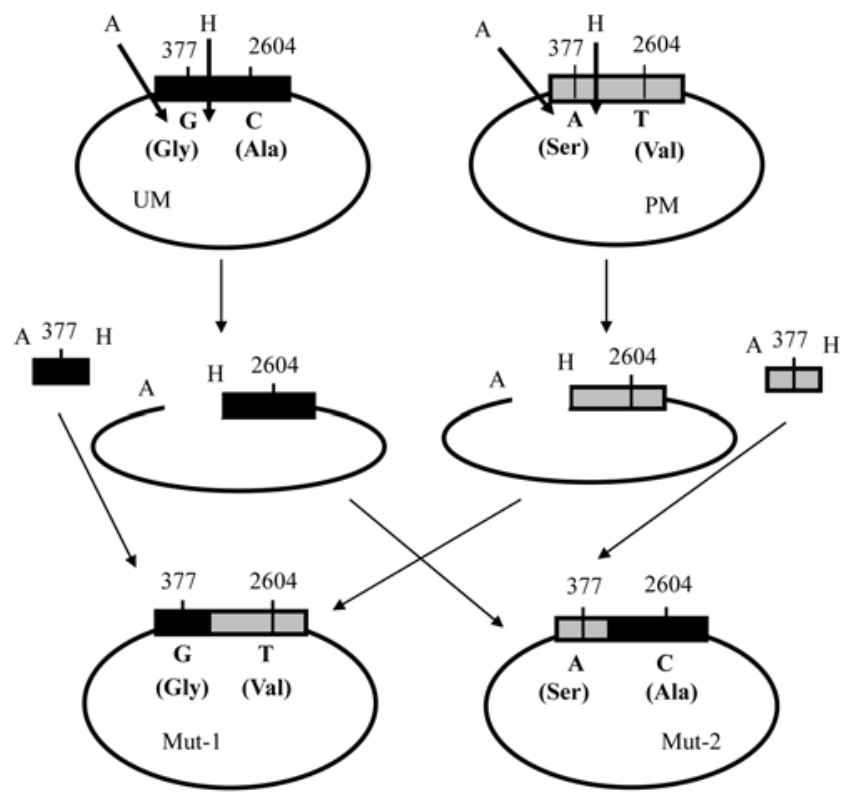

Fig. 1. Construction strategies for mutant plasmids of $A O$ in Donryu strain rats. The chimeric cDNAs, Mut-1 and Mut-2, were constructed by restriction enzyme treatment and ligation in such a way that they had the substituted nucleotide sequences of $(377 \mathrm{G}$, 2604T), and (377A, 2604C), respectively. The restriction sites were shown as A for Acc65I and $\mathrm{H}$ for $H p a \mathrm{I}$, respectively.

(377G, 2604T) and Mut-2 had those of (377A, 2604C). The transfection into $E$. coli XL1-Blue MRF' and the insertion check of each plasmid were performed as described above.

Cell cultures. The four kinds of AO cDNA plasmids purified using Wizard Plus Minipreps DNA Purification Systems were transfected into the expression E. coli M15 cells (pREP4, QIAGEN). The control sample was produced by the transfection of the pQE-30 Xa vector. A single colony was cultured at $37^{\circ} \mathrm{C}$ overnight in $5 \mathrm{ml}$ of LB medium containing $100 \mu \mathrm{g} / \mathrm{ml}$ ampicillin and $25 \mu \mathrm{g} / \mathrm{ml}$ kanamycin. To the overnight culture, $500 \mathrm{ml}$ of LB with $100 \mu \mathrm{g} / \mathrm{ml}$ ampicillin and $25 \mu \mathrm{g} / \mathrm{ml}$ kanamycin were added and cultured at $37^{\circ} \mathrm{C}$ until absorbance at $600 \mathrm{~nm}$ reached 0.5 0.6. Then, IPTG $(1 \mathrm{mM})$ was added to the mixture along with ATP $(1 \mathrm{mM})$, riboflavin $(3 \mu \mathrm{M})$, and $\mathrm{Na}_{2} \mathrm{MoO}_{4}(50 \mu \mathrm{M})$ according to the methods already reported (Huang et al., 1999). Growth of the culture was allowed to continue for $72 \mathrm{~h}$ at $22^{\circ} \mathrm{C}$. After collection by centrifugation at 7,000 $\mathrm{g}$ for 10 min at $4^{\circ} \mathrm{C}$, the cells were solubilized with QIAexpressionist (QIAGEN) according to the manufacturer's instructions. The soluble proteins were applied to a HisTrap HP Column (GE Healthcare Ltd.) fully pre-equilibrated with $10 \mathrm{mM}$ phosphate buffer ( $\mathrm{pH}$ 7.4) containing $20 \mathrm{mM}$ imidazole and $0.5 \mathrm{M} \mathrm{NaCl}$ (Buffer A). After being washed with $100 \mathrm{mM}$ imidazole in Buffer $\mathrm{A}$, the target enzyme was eluted with $250 \mathrm{mM}$ imidazole in Buffer A.

Sodium dodecyl sulfate polyacrylamide gel electrophoresis (SDS-PAGE). The expressed enzymes were separated by sodium dodecyl sulfate-polyacrylamide gel electrophoresis (SDS-PAGE), which was performed using PhastGel gradient 4-15 in PhastGel SDS Buffer Strips (GE Healthcare). An automatic electrophoresis
Table 1. Analysis of genomic DNA and amino acid substitution of AO in UM, EM, and PM groups of Donryu strain rats

\begin{tabular}{cccc}
\hline $\begin{array}{c}\text { Position of nucleotide } \\
\text { or amino acid }\end{array}$ & UM & EM & PM \\
\hline $\begin{array}{c}\text { Nucleotide } 377 \\
\text { Amino acid 110 }\end{array}$ & G & G/A & A \\
Nucleotide 2604 & C & C/T & Ser \\
Amino acid 852 & Ala & Ala/Val & Val \\
\hline
\end{tabular}

The number of rats examined for respective ultrarapid metabolizer (UM), extensive metabolizer (EM), and poor metabolizer (PM) with regard to the AO-catalyzed 2-oxidation activity of (S)-RS-8359 was ten for each type. The nucleotide sequences at 377 and 2604 positions were completely consistent within a respective phenotype group. The nucleotide and amino acid was homozygous in the UM and PM rats, but heterozygous in the EM rats.

system, PhastSystem (GE Healthcare), was used. Protein bands were stained with Coomassie Brilliant Blue CBB R-350 (GE Healthcare). An HMW Calibration Kit and HMV Native Marker Kit (GE Healthcare) were used for molecular mass standards.

Statistical analysis. The results are expressed as the mean $\pm \mathrm{SE}$ for the number of experiments. Statistical significance between low and high activity groups was compared by a Student's $t$-test. Values with $p<0.05$ were considered statistically significant.

\section{Results}

Genomic DNA analysis. The genomic DNA of livers from the UM, EM and PM Donryu strain rats was analyzed by the direct sequence method to determine whether the nucleotide mutation observed in the cDNA is also present in the genomic DNA. The nucleotide sequences were $(377 \mathrm{G}, 2604 \mathrm{C})$ in the UM group, $(377 \mathrm{G} / \mathrm{A}, 2604 \mathrm{C} / \mathrm{T})$ in the EM group, and (377A, 2604T) in the PM group, thus being homozygous in the UM and PM groups, but heterozygous in the EM group (Table 1). The results of the genomic DNA were in complete accord with those of the cDNA.

Construction of expression system for naturally existing and mutant AO cDNA. AO cDNAs of UM and PM in Donryu strain rats were introduced into the expression vector pQE-30 Xa. The chimeric cDNA plasmids of Mut-1 having (377G, 2604T) and Mut-2 having (377A, 2604C) were constructed by cleavage of pQE-30 Xa with restriction enzymes Acc65I/ HpaI followed by cross-ligation of the fragments obtained. The introductions of the objective nucleotides were confirmed by sequence analysis, as shown in Fig. 2.

SDS-PAGE. Each of the constructed plasmids of the UM, PM, Mut-1, and Mut-2 groups was transfected into the expression E. coli M15 cells. The solubilized supernatant of the expressed enzymes was analyzed by SDS-PAGE. All of 


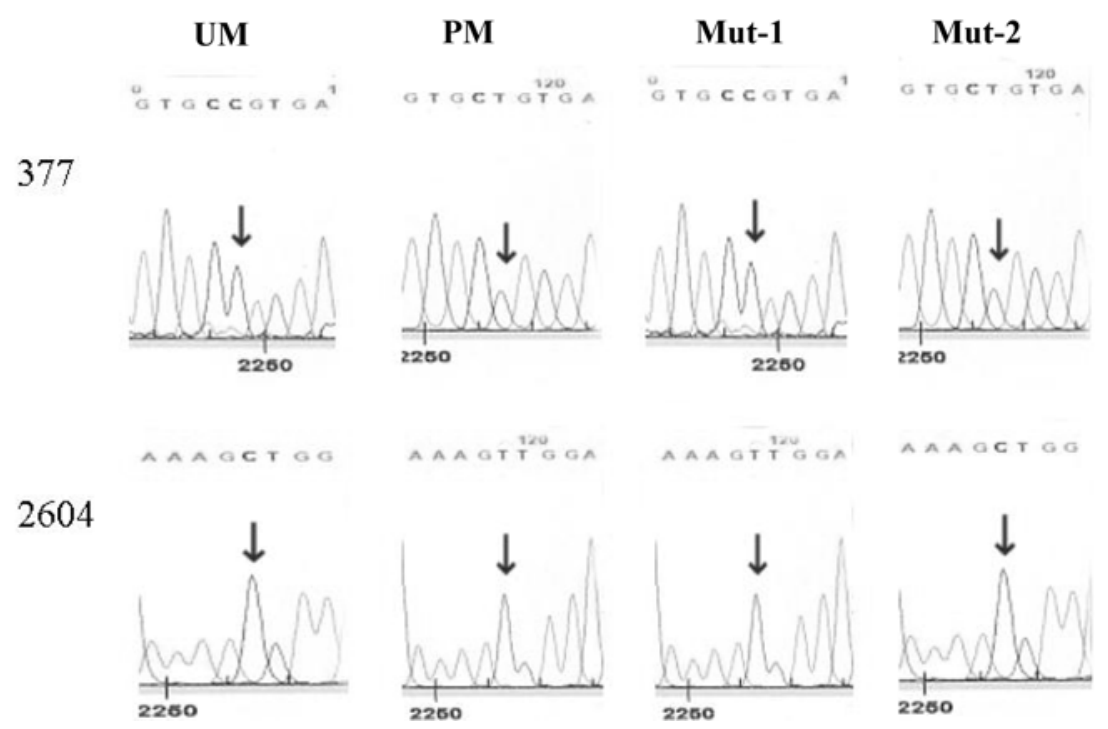

Fig. 2. Sequence analysis at positions 377 and 2604 of AO cDNA from naturally existing UM and PM, and from mutants Mut-1 and Mut-2. The construction strategies used in the current study were shown to result in point mutation at either position 377 or 2604 of UM and PM.

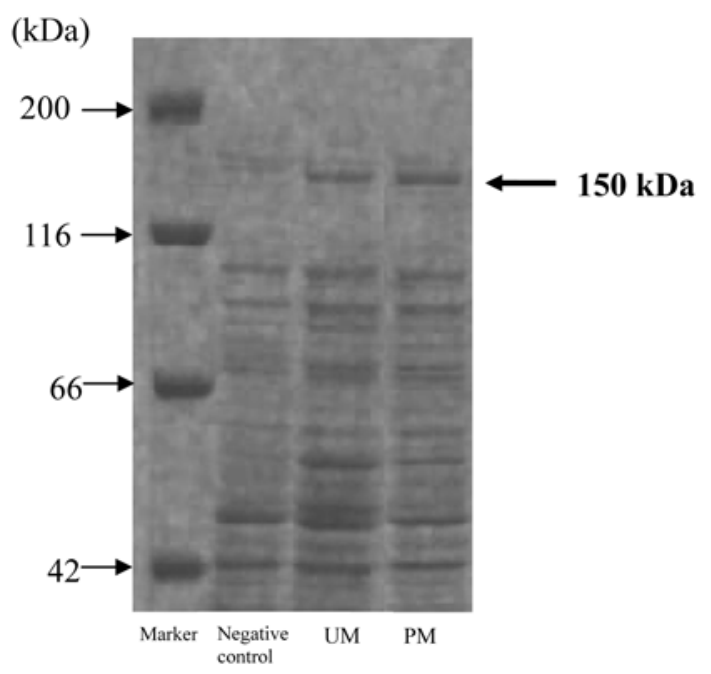

Fig. 3. SDS-PAGE of AO protein expressed in M15 cells. SDSPAGE was performed using PhastGel gradient 4-15 in PhastGel SDS Buffer Strips; the proteins on the gel were stained with Coomassie Brilliant Blue.

them displayed the target protein at a high concentration that was not detected in the control supernatant sample (Fig. 3).

Analysis of kinetic parameters for expressed enzymes. The AO-catalyzed 2-oxidation activities of $(S)$-RS-8359 by the enzymes expressed in $E$. coli were measured. The kinetic parameters calculated from Hanes-Woolf plots (Fig. 4) are summarized in Table 2. A significant difference of several fold in $K_{m}$ values was seen between UM and the other groups. The expressed AO enzymes of the UM and PM groups exhibited high and low activity, respectively. Thus, the genetic polymorphism of AO activities in Donryu strain rats observed in liver cytosol was also confirmed in the expression system. Mut-1 and Mut-2 showed almost the same high and low $V_{\max }$ values as that of the UM and PM groups, respectively.

\section{Discussion}

During the study of rat strain differences in the AO-catalyzed 2-oxidation of RS-8359 (25), which is an MAO-A inhibitor (Yokoyama et al., 1989; Kumagae et al., 1991; Miura et al., 1993; Iwata et al., 1996) and has been developed as an antidepressant (Puchler et al., 1997; Plenker et al., 1997), we were aware of the individual variations in the activity in Donryu strain rats. Analysis of the AO cDNA revealed that the variations were due to genetic polymorphism in that UM possessed the nucleotide mutation of (377G, 2604C) coding for amino acid substitution of (110Gly, 852Ala), EM with (377G/A, 2604C/T) coding for (110Gly/Ser, 852Ala/Val), and PM with (377A, 2604T) coding for (110Ser, 852Val) (Itoh et al., 2007). The same nucleotide mutations were now confirmed to also be present in the genomic DNA. The results suggested that the nucleotide $377 \mathrm{G}$ or $2604 \mathrm{~T}$ is primarily important for maintaining the catalytic activity of AO. The study using the expression system of mutant AO cDNA should be the best method of elucidating which nucleotide mutation plays the most important role in the polymorphism of AO in Donryu strain rats.

Although the expression of catalytically active molybdoflavoenzymes in prokaryotes has been known to be generally difficult (Garattini et al., 2003), Huang et al. (1999) has for the first time presented findings that mouse retinal oxidase (aldehyde oxidase) was expressed in an active form in a prokaryotic system, E. coli BL21, in which riboflavin, ATP, and $\mathrm{Na}_{2} \mathrm{MoO}_{4}$ were added. Based on these findings, we tried 
(A) $\mathbf{U M}$

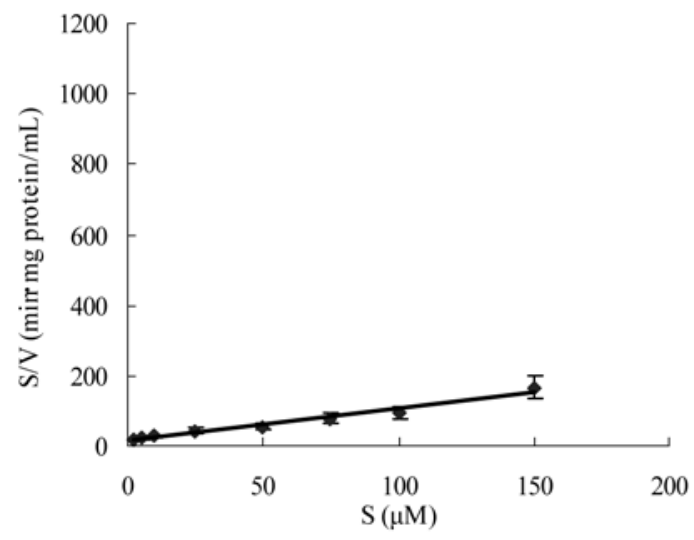

(C) Mut-1

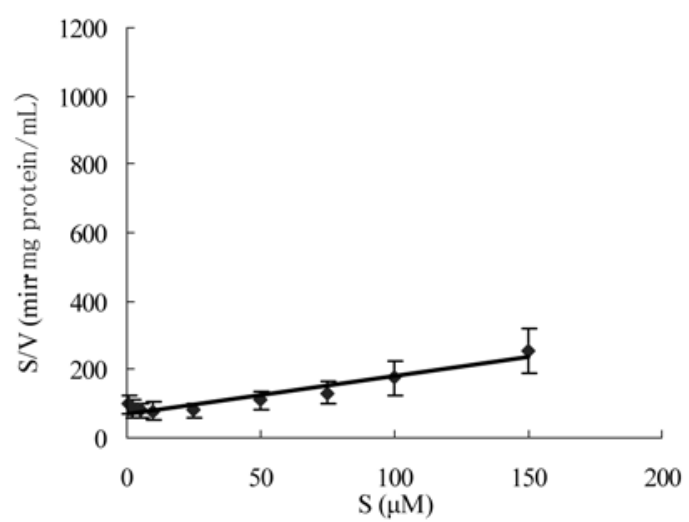

(B) PM

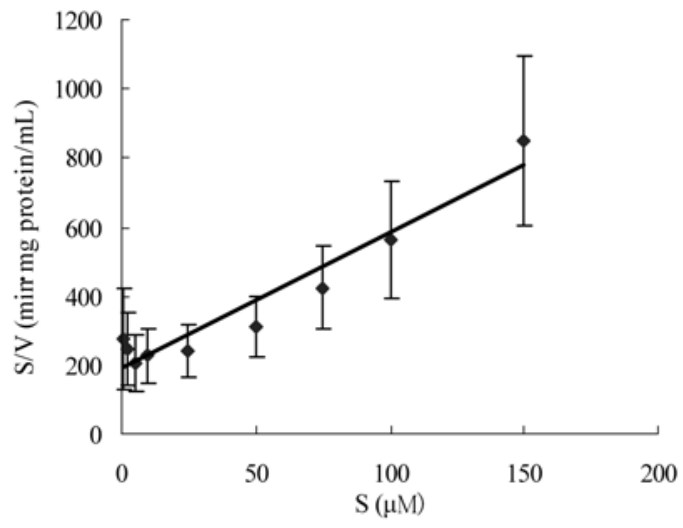

(D) Mut-2

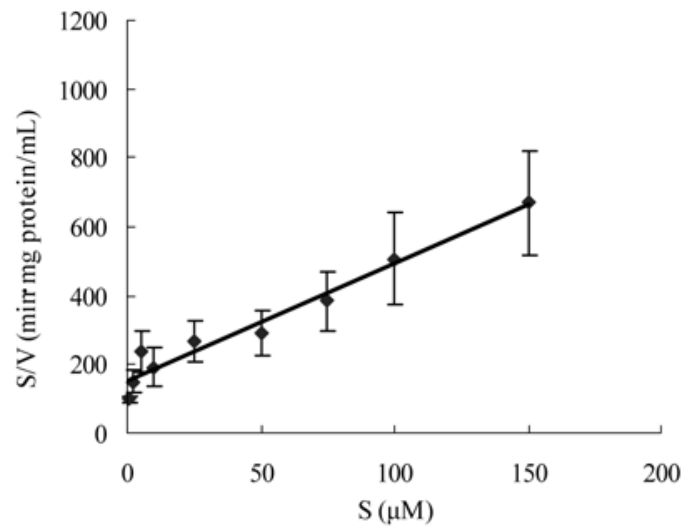

Fig. 4. Hanes-Woolf plots for the AO-catalyzed 2-oxidation of (S)-RS-8359 by naturally occurring and expressed AO proteins. A, UM; B, PM; C, Mut-1; D, Mut-2. The 2-oxidation of $(S)$-RS-8359 was determined in three experiments over a substrate concentration range of 1.0 to $150 \mu \mathrm{M}$.

to express rat and monkey $\mathrm{AO}$ protein in E. coli strain M15 that is different from the previously reported strain. As a result, the AO protein was clearly detected on SDS-PAGE/ Western blot analysis, and the expressed monkey $\mathrm{AO}$ exhibited a biphasic Eadie-Hofstee profile for the 2-oxidation of $(S)-\mathrm{RS}-8359$, but the rat AO did not. The results were in

Table 2. Kinetic parameters for the 2-oxidation of (S)-RS-8359 by AO protein expressed in $E$. coli M15 cells

\begin{tabular}{ccc}
\hline Expression type & $\begin{array}{c}K_{m} \\
(\mu \mathrm{M})\end{array}$ & $\begin{array}{c}V_{\max } \\
(\mathrm{nmol} / \mathrm{min} / \mathrm{mg} \text { protein })\end{array}$ \\
\hline UM (5) & $16.0 \pm 2.40$ & $1.26 \pm 0.26$ \\
Mut-1 (5) & $62.4 \pm 6.98^{*}$ & $1.20 \pm 0.32$ \\
Mut-2 (4) & $44.3 \pm 5.34^{*}$ & $0.36 \pm 0.09^{*}$ \\
PM (4) & $36.4 \pm 4.54^{*}$ & $0.32 \pm 0.10^{*}$ \\
\hline
\end{tabular}

Results are expressed as the mean \pm S.E. of four (Mut- 2 and PM) or five (UM and Mut-1) determinations. The nucleotide sequence at 377 and 2604 are $\mathrm{G}$ and $\mathrm{C}$ in the UM group, $\mathrm{G}$ and $\mathrm{T}$ in the Mut-1 group, $\mathrm{A}$ and $\mathrm{C}$ in the Mut-2 group, and $\mathrm{A}$ and $\mathrm{T}$ in the PM group, respectively. ${ }^{*} p<0.05$ compared with the UM group. good agreement with those obtained in the liver cytosol (Hoshino et al., 2007). Recently, Yamaguchi et al. (2007) has also reported the expression of human xanthine oxidoreductase (XOR) and its mutants in E. coli strain JM109.

In this report, plasmids Mut-1 with (377G, 2604T) and Mut-2 with (377A, 2604C) were constructed and expressed in E. coli M15 as well as the naturally occurring UM with (377G, 2604C) and PM with (377A, 2604T). The expressed UM and PM enzymes showed high and low AO activity, respectively, thus the individual differences observed in liver cytosol were also reproduced in the expression system. Mut-1 and Mut-2 demonstrated almost the same $V_{\max }$ values as did those of UM and PM, respectively. The $K_{m}$ value of UM was one-fourth of those of the other groups and was significantly smaller, although the reason is unclear. However, the difference did not exceed one order of magnitude, suggesting that it might not be large enough to warrant extended discussion. Although the number involved in the ratio in liver cytosolic extracts was only two samples ( 1 male and 1 female), the ratio between the $V_{\max }$ values for the UM as compared to the PM phenotypes was an order of magnitude greater than those found in the in vitro expressed proteins. Some in vitro and in vivo reasons are 
imaginable. The ratio calculated from cytosol data (Itoh et al., 2007) might be overestimated by using a crude enzyme that contains a large amount of contaminated proteins. In fact, significantly more AO was expressed in EM rat liver compared to the PM group. The UM group showed a tendency to have a much higher AO concentration than did the PM group (data not shown because the sample consisted of only two rats). If AO protein is purified from both the PM and UM rats and the activity is expressed per mg protein, the discrepancy might be reduced. Additionally, there might be some important posttranslational modification to affect the AO activity of the PM or UM rats in vivo. Furthermore, the employment of HisTag for easy and effective purification of the expressed protein might differently affect the expression of the PM and UM cDNA. Regardless of these and other possibilities, the actual reason is still unknown. However, the expression results were at least likely to reflect the cytosolic results qualitatively.

Taken together, the results indicated that the genetic polymorphism of AO activity in Donryu strain rats is possibly caused by the nucleotide mutation of $377 \mathrm{G}>\mathrm{A}$, but not by $2604 \mathrm{C}>\mathrm{T}$. The $377 \mathrm{G}>\mathrm{A}$ nucleotide mutation results in the amino acid substitution from a symmetric Gly to an asymmetric Ser at position 110 near the second Fe-S cluster (Wright et al., 1999). The amino acid substitution might provoke a serious conformational change or a posttranslational modification of AO protein to decrease the catalytic activity significantly. It might be helpful to study the physicochemical properties of the proteins expressed by the PM and Mut- 2 cDNAs to reveal the mechanistic reasons for the Gly ${ }^{110}$ Ser amino acid substitution affecting the AO structure and its activity. The same genetic reason might apply for the rat strain differences in $\mathrm{AO}$ activity. That is one of the subjects we are now investigating to clarify the molecular mechanism of individual differences in $\mathrm{AO}$ activity in Donryu rats and strain differences in rats.

In addition to AOX1 and xanthine oxidase, the presence of aldehyde oxidase homolog 1 and 2 (AOH1 and $\mathrm{AOH} 2$ ) has been described as a member of the molybdenum hydroxylases (Terao et al., 2000, 2001; Vila et al., 2004; Kurosaki et al., 2004). The homologs were first known only in mice, but recently were also found in rats. Furthermore, a novel molybdo-flavoenzyme, $\mathrm{AOH} 3$, has been identified in both mice and rats. Among the $\mathrm{AO}$ isoforms, $\mathrm{AOH} 1$ is expressed in liver and shows a similar substrate specificity as that of AOX1, but AOX1 is generally more efficient than AOH1. Although the properties of $\mathrm{AOH} 1$ have been well characterized in mice, those of rat $\mathrm{AOH} 1$ have not always been clarified in detail other than cDNA cloning. Thus, many properties are still unclear such as substrate specificity, inhibitor susceptibility, molecular mass, tissue distribution, regulation by sex hormone, strain differences, species differences, and so on. Under these conditions, we investigated polymorphic variation in the $(S)$ RS-8359 2-oxidation activity in Donryu strain rats from the viewpoint of nucleotide mutation of AO (Itoh et al., 2007). It was found that $\mathrm{AO}$ phenotypes were clearly consistent with nucleotide mutations coding for amino acid substitutions of
Gly ${ }^{110}$ Ser. However, the presence of AOH1 having similar properties as AOX1 in mice suggests the possibility of participation of $\mathrm{AOH} 1$ mutation in the genetic polymorphism seen in Donryu strain rats. This issue should be an interesting subject for future study.

In conclusion, the $377 \mathrm{G}>\mathrm{A}$ nucleotide mutation was directly proved to be important for the polymorphism of $\mathrm{AO}$ in Donryu strain rats by using the expression systems of mutant AO cDNAs.

Acknowledgments We wish to express our thanks to Dr. T. Ikeda, Director of the Drug Metabolism and Pharmacokinetics Research Laboratories, Daiichi-Sankyo Co. Ltd., for his kind encouragement.

\section{References}

Beedham, C. (1985) Molybdenum hydroxylases as drug-metabolizing enzymes. Drug Metab. Rev. 16, 119-156.

Beedham, C. (1987) Molybdenum hydroxylases: Biological distribution and substrate-inhibitor specificity. Prog. Med. Chem. 24, 85-121.

Beedham, C. (1997) The role of non-P450 enzymes in drug oxidation. Pharm. World Sci. 19, 255-263.

Beedham, C. (1998) Molybdenum hydroxylases; in Metabolism of Xenobiotics, Gorrod, J. W., Oeschlager, H., and Caldwell, J. (eds), pp. 51-58, Taylor and Francis, London and New York, USA.

Beedham, C. (2002) Molybdenum hydroxylase; in Enzyme Systems that Metabolise Drug and Other Xenobiotics, Ioannides, C. (ed.), pp. 147-187, John Wiley, Chichester, UK.

Beedham, C., Miceli, J. J. and Obach, S. (2003) Ziprasidone metabolism, aldehyde oxidase, and clinical implications. J. Clin. Psychopharm. 23, 229-232.

Glueksohn-Waelsch, S., Greengard, P., Quinn, G. P. and Teicher, L. S. (1967) Genetic variations of an oxidase in mammals. J. Biol. Chem. 242, 1271-1273.

Huang, D. Y., Furukawa, A. and Ichikawa, Y. (1999) Molecular cloning of retinal oxidase/aldehyde oxidase cDNA from rabbit and mouse liver and functional expression of recombinant mouse retinal oxidase cDNA in Escherichia coli. Arch Biochem. Biophys. 364, 264-272.

Itoh, K., Yamamura, M., Muramatsu, S., Hoshino, K., Masubuchi, A., Sasaki, T. and Tanaka, Y. (2005) Stereospecific oxidation of (S)-enantiomer of RS-8359, a selective and reversible MAO-A inhibitor, by aldehyde oxidase. Xenobiotica 35, 561-573.

Itoh, K., Yamamura, M., Takasaki, W., Sasaki, T., Masubuchi, A. and Tanaka, Y. (2006) Species differences in enantioselective 2oxidation of RS-8359, a selective and reversible MAO-A inhibitor, and cinchona alkaloids by aldehyde oxidase. Biopharm. Drug Dispos. 27, 1133-1139.

Itoh, K., Masubuchi, A., Sasaki, T., Adachi, M., Watanabe, N., Nagata, K., Yamazoe, Y., Hiratsuka, M., Mizugaki, M. and Tanaka, Y. (2007) Polymorphism of aldehyde oxidase in Donryu rats. Drug Metab. Dispos. 35, 734-739.

Iwata, N., Tonohiro, T., Kozuka, M., Kumagae, Y., Takasaki, W. and Tanaka, Y. (1996) A novel selective and reversible MAO-A inhibitor, RS-8359: its pharmacological properties and 
metabolism. Int. Acad. Biomed. Drug Res. 11, 285-286.

Jordan, C. G. M., Rashidi, M. R., Laljee, H., Clarke, S. E., Brown, J. E. and Beedham, C. (1999) Aldehyde oxidase-catalyzed oxidation of methotrexate in the liver of guinea pig, rabbit and man. J. Pharm. Pharmacol. 51, 411-418.

Kitamura, S., Sugihara, K., Nakatani, K., Ohta, S., O’Hara, T., Nimomiya, S., Green, C. E. and Tyson, C. A. (1999a) Variation of hepatic methotrexate 7-hydroxylase activity in animals and humans. IUBMB Life. 48, 607-611.

Kitamura, S., Nakatani, K., Sugihara, K., and Ohta, S. (1999b) Strain differences of the ability to hydroxylate methotrexate in rats. Com. Biochem. Phys. Part C 122, 331-336.

Kitamura, S., Sugihara, K. and Ohta, S. (2006) Drug-metabolizing ability of molybdenum hydroxylases. Drug Metab. Pharmacokinet. 21, 83-98.

Kumagae, Y., Matsui, Y. and Iwata, N. (1991) Deamination of norepinephrine, dopamine, and serotonin by type A monoamine oxidase in discrete regions of the rat brain and inhibition by RS8359. Jpn. J. Pharmacol. 55, 121-128.

Miura, H., Naoi, M., Nakahara, D., Ohta, T. and Nagatsu, T. (1993) Changes in monoamine levels in mouse brain elicited by forcedswimming stress, and the protective effect of a new monoamine oxidase inhibitor, RS-8359. J. Neural Transm. 94, 175-187.

Plenker, A., Puchler, K. and Volz, H. P. (1997) The effects of RS8359 on cardiovascular function in healthy subjects and depressed patients. Int. Clin. Psychopharm. 12, S25-S29.

Prakash, C., Kamel, A., Gummerus, J. and Wilner, K. (1997) Metabolism and excretion of a new antipsychotic drug, ziprasidone, in humans. Drug Metab. Dispos. 25, 863-875.

Puchler, K., Schaffler, K. and Plenker, A. (1997) The comparative effects of single and multiple doses of RS-8359, moclobemide and placebo on psychomotor function in healthy subjects. Int. Clin. Psychopharm. 12, 17-23.

Rashidi, M. R., Smith, J. A., Clarke, S. E. and Beedham, C. (1997) In vitro oxidation of famciclovir and 6-deoxypenciclovir by aldehyde oxidase from human, guinea pig, rabbit and rat liver. Drug Metab. Dispos. 25, 805-813.

Sasaki, T., Masubuchi, A., Yamamura, M., Watanabe, N., Hiratsuka, M., Mizugaki, M., Itoh, K. and Tanaka, Y. (2006) Rat strain differences in stereospecific 2-oxidation of RS-8359, a reversible and selective MAO-A inhibitor, by aldehyde oxidase. Biopharm. Drug Dispos. 27, 247-255.

Sugihara, K., Kitamura, S. and Tatsumi, K. (1995) Strain differences of liver aldehyde oxidase in rats. Biochem. Mol. Biol. Int. 37, 861869.

Takasaki, W., Yamamura, M., Nozaki, A., Nitanai, T., Sasahara, K., Itoh, K. and Tanaka, Y. (2005) Stereoselective pharmacokinetics of RS-8359, a selective and reversible MAO-A inhibitor, by species-dependent drug metabolizing enzymes. Chirality 17, 135141.

Wright, R. M., Clayton, D. A., Riley, M. G., McManaman, J. L. and Repine, J. E. (1999) cDNA cloning, sequencing, and characterization of male and female rat liver aldehyde oxidase (rAOX1). J. Biol. Chem. 274, 3878-3886.

Yamaguchi, Y., Matsumura, T., Ichida, K., Okamoto, K. and Nishino, T. (2007) Human xanthine oxidase changes its substrate specificity to aldehyde oxidase type upon mutation of amino acid residues in the active site: role of active site residues in binding and activation of purine substrate. J. Biochem. 141, 513-524.

Yokoyama, T., Karube, T. and Iwata, N. (1989) Comparative studies of the effects of RS-8359 and safrazine on monoamine oxidase in vitro and in vivo in mouse brain. J. Pharm. Pharmacol. 41, 32-36. 